\title{
Correction to: Tilorone: a Broad-Spectrum Antiviral Invented in the USA and Commercialized in Russia and beyond
}

Sean Ekins ${ }^{\prime} \cdot$ Thomas R. Lane' • Peter B. Madrid ${ }^{2}$

Published online: 6 November 2020

(C) Springer Science+Business Media, LLC, part of Springer Nature 2020

\section{Correction to: Pham Res (2020) 37: 7 I \\ https://doi.org/10.1007/s | 1095-020-02799-8}

Tables 1 and 2 had data in the bottom half (right hand side) of each table that was formatted incorrectly in the final proof version versus the submitted version.

The original article has been corrected.

The online version of the original article can be found at https://doi.org/l 0 . | 007/s | | 095-020-02799-8

Sean Ekins

sean@collaborationspharma.com

Collaborations Pharmaceuticals, Inc., 840 Main Campus Drive, Lab 35I0, Raleigh, NC 27606, USA

2 SRI International, 333 Ravenswood Avenue, Menlo Park, CA 94025, USA 
Table I Literature data on Tilorone antiviral activity

\begin{tabular}{|c|c|c|c|c|}
\hline Virus & Genus & Type & Efficacy & Reference \\
\hline Mengo virus & cardiovirus & + ssRNA & $80 \%$ survival in mice at $250 \mathrm{mg} / \mathrm{kg}$ single oral dose & $(\mid 2)$ \\
\hline Semliki Forest & alphavirus & + ssRNA & $100 \%$ survival in mice at $250 \mathrm{mg} / \mathrm{kg}$ single oral dose & $(\mid 2)$ \\
\hline Vesicular stomatitis & vesiculovirus & -ssRNA & $20 \%$ survival but delayed time to death with two $250 \mathrm{mg} / \mathrm{kg}$ doses & $(\mid 2)$ \\
\hline Encephalomyocarditis virus (EMCV) & cardiovirus & + ssRNA & $80 \%$ survival in mice at $250 \mathrm{mg} / \mathrm{kg}$ single oral dose & $(12)$ \\
\hline Influenza B & Betainfluenza virus & -ssRNA & $50 \%$ survival in mice at $250 \mathrm{mg} / \mathrm{kg}$ single oral dose & $(\mid 2)$ \\
\hline Influenza A/Equine- I & Alphainfluenza virus & -ssRNA & $40 \%$ survival in mice at $250 \mathrm{mg} / \mathrm{kg}$ single oral dose & $(12)$ \\
\hline Influenza A/Jap/305 & Alphainfluenza virus & -ssRNA & $30 \%$ survival in mice at $250 \mathrm{mg} / \mathrm{kg}$ single oral dose & $(\mid 2)$ \\
\hline Herpes simplex virus I & simplexvirus & dsDNA & $45 \%$ survival in mice at $250 \mathrm{mg} / \mathrm{kg}$ oral doses for 7 days & $(\mid 2)$ \\
\hline West Nile virus & flavivirus & + ssRNA & $46 \%$ lethality protection at $10 \mathrm{mg} / \mathrm{kg}$ & $(19)$ \\
\hline MERS & Betacoronavirus & + ssRNA & $\mathrm{EC}_{50} 10.56 \mu \mathrm{M}, \mathrm{CC}_{50}>20 \mu \mathrm{M}$ & $(\mid 8)$ \\
\hline VEEV & Alphavirus & + ssRNA & I log drop of virus titer at $25 \mathrm{mg} / \mathrm{ml}$ in vitro & $(20)$ \\
\hline EMCV & cardiovirus & + ssRNA & I log drop of virus titer at $25 \mathrm{mg} / \mathrm{ml}$ in vitro & $(20)$ \\
\hline VEEV & Alphavirus & + ssRNA & I00\% survival in mice at $250 \mathrm{mg} / \mathrm{kg}$ & $(21)$ \\
\hline
\end{tabular}

Table 2 Recent antiviral screening data for Tilorone generated under the NIAID-DMID NCEA antiviral in vitro screening services except where noted

\begin{tabular}{|c|c|c|c|c|c|c|c|}
\hline Virus & Strain & Genus & Type & Cell line & $\mathrm{EC}_{50}(\mu \mathrm{M})$ & $\mathrm{CC}_{50}(\mu \mathrm{M})$ & Reference \\
\hline EBOV & Zaire & Filovirus & -ssRNA & HeLa & 0.23 & 6.2 & $(31)$ \\
\hline EBOV & Zaire & Filovirus & - ssRNA & Vero 76 & $>||^{*}$ & $\mid 1$ & (32) \\
\hline MARV & Musoke & Filovirus & - ssRNA & HeLa & 1.9 & - & (33) \\
\hline Influenza A virus HINI & California/07/20/09 & Influenzavirus & - ssRNA & MDCK & $>19$ & 19 & \\
\hline Tacaribe virus & TRVL I I573 & Arenavirus (new world) & $-/+$ ssRNA & Vero 76 & $29 *$ & 32 & \\
\hline $\mathrm{CHIKV}$ & S27 (VR-67) & Alphavirus & + ssRNA & Vero 76 & $4.2^{*}$ & 32 & (34) \\
\hline MERS-CoV & EMC & Betacoronavirus & + ssRNA & Vero 76 & $3.7^{*}$ & 36 & (34) \\
\hline Poliovirus 3 & WM-3 & Enterovirus & + ssRNA & Vero 76 & $>25^{*}$ & 25 & \\
\hline VEEV & TC-83 & Alphavirus & + ssRNA & Vero 76 & | 8* & 32 & \\
\hline
\end{tabular}

*In vitro antiviral data in Vero 76 cells may underestimate antiviral activity due to lacking IFN pathways 